\title{
Urethral cultures in female patients with a spinal cord injury
}

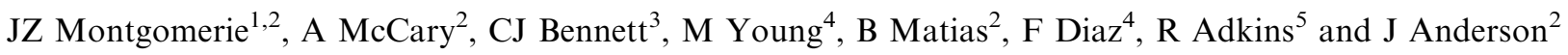 \\ ${ }^{1}$ Professor of Medicine, USC School of Medicine, University of Southern California, Los Angeles, CA; ${ }^{2}$ Department \\ of Medicine, Rancho Los Amigos Medical Centre, Downey, CA; ${ }^{3}$ Associate Professor of Urology, USC School of \\ Medicine, University of Southern California, Los Angeles CA and Chief, Urology, Rancho Los Amigos Medical \\ Center, Downey, CA; ${ }^{4}$ Urological Nursing Services, Department of Nursing, Rancho Los Amigos Medical Center, \\ Downey, CA; ${ }^{5}$ Director Spinal Cord Injury Project of Southern California, Rancho Los Amigos Medical Center, \\ Downey, CA, USA
}

\begin{abstract}
Quantitative cultures of the urethral meatus were obtained from women with SCI undergoing intermittent catheterization. When compared with the urethral cultures of a group of female subjects, women with SCI had a greater number of isolates of Klebsiella pneumonia and Pseudomonas aeruginosa in the urethral flora. However there was not a significantly greater number of isolates or $\log$ numbers of E. coli or Enterococcus sp. in the urethral flora. The $E$. coli and Enterococcus sp. isolated from the urine were not isolated from the urethra of female patients with SCI in one third of the patients. This poor correlation between the simultaneous urethral and urine cultures of female subjects with SCI may reflect colonization of the urine with organisms that were unable to adhere to the mucosa and colonize the urethra. To what extent these organisms colonize or are temporary residents may be important in the pathogenicity of the infection.
\end{abstract}

Keywords: urethral colonization; urinary tract infections; female; spinal cord injury; E. coli; Enterococcus

\section{Introduction}

Urinary tract infections occur in women with spinal cord injury as a result of the neuropathic bladder, with the bacteria usually being introduced into the bladder at the time of intermittent catheterization. ${ }^{1}$ The immediate source of the bacteria causing infections is assumed to be the flora in the periurethral area. Women with spinal cord injury while in hospital during their rehabilitation may have changes in the urethral flora because of a number of factors.

As part of a study comparing the MMG/O'Neill catheter (Medical Marketing Group, Atlanta, Georgia), with the standard catheter we examined the urethral flora prior to the development of bacteriuria. The total aerobic flora, the aerobic gram negative bacilli, and the Enterococcal species in the urethral flora of female patients with SCI were measured during their initial rehabilitation in hospital. Urine cultures were obtained at the same time to determine the relation of the urethral cultures to bacteriuria. The results of the urethral cultures were compared with those of hospital personnel without evidence of disease of the urinary tract.

Correspondence: John Z Montgomerie MD, Infectious Disease Division, Department of Medicine, Rancho Los Amigos Medical Center, 7601 East Imperial Highway, Downey, CA 90242, USA

\section{Methods}

Cultures of the urethral meatus of women with SCI on intermittent catheterization were taken at weekly intervals until the patients developed significant bacteriuria $(>10,000 / \mathrm{ml})$ and were treated with antibiotics. Significant bacteriuria was treated regardless of symptoms. At that time the patients were removed from the study until they no longer had bacteriuria and were off antibiotics. Quantitative cultures of swabs of the urethral meatus were used to determine the total aerobic bacteria and the log number of isolates of gram negative aerobic bacilli (GNB) and Enterococcus. The results of the cultures were compared with those of normal female subjects (hospital personnel).

Urethral cultures in women were obtained using sterile cotton swabs, moistened with sterile saline. Cultures were obtained before the patients were prepared for intermittent catheterization and the collection of the urine for culture. The swabs were inserted approximately $1 \mathrm{~cm}$ into the urethra. Swabs were placed in normal saline $(2 \mathrm{ml})$ and refrigerated at $4{ }^{\circ} \mathrm{C}$ until plated within $2 \mathrm{~h}$. Swabs were agitated with the vortex and 0.01 and $0.1 \mathrm{ml}$ was plated onto selective media for Pseudomonas sp., (Pseudomonas Isolation Agar, Difco Laboratories, Detroit, Mich.) and Klebsiella sp. ${ }^{2}$ The cultures were also plated on MacConkey media to detect E. coli and other gram 
negative aerobic bacilli, CNA for enterococcus species and BHIA to determine total number of aerobic bacteria.

\section{Results}

Isolation of Gram negative bacilli and Enterococcus sp. from the urethra of female SCI patients and controls The number of isolates of gram negative bacilli and Enterococcus sp. from each group is shown in Table 1. Forty-eight cultures were obtained from 17 female SCI patients. The mean number of cultures from each patient was 2.8 (range 1-7). A single culture was obtained from the control group of 19 normal women. A disproportionate number of Klebsiella sp. and Pseudomonas sp. were isolated from the patients with SCI. E. coli and Enterococcus sp. were the dominant flora in these cultures but the proportion of individuals from whom Enterococci and E. coli were isolated was not significantly greater in women with SCI than female controls when those patients with bacteriuria were excluded (Table 2). In addition the log number of E. coli and Enterococcus sp. in the urethral flora was not significantly greater in women with SCI than female controls (Table 3) when cultures obtained at the time of bacteriuria were excluded.

Relation of Gram negative bacilli and Enterococcus $s p$. in urethra and urine of female patients with SCI

The results of the cultures of the urine and the urethra at the time the patients were found to have developed

Table 1 Gram negative bacteria and Enterococcus in the urethra

\begin{tabular}{lcc}
\hline Bacteria & $\begin{array}{c}\text { Patients } \\
(n=48)\end{array}$ & $\begin{array}{c}\text { Female controls } \\
(n=19)\end{array}$ \\
\hline E. coli & 17 & 5 \\
Klebsiella & 12 & 2 \\
Pseudomonas & 5 & 0 \\
Proteus sp. & 3 & 1 \\
Enterobacter sp. & 2 & 1 \\
Citrobacter sp. & 2 & 2 \\
Acinetobacter sp. & 0 & 1 \\
Enterococcus sp. & 17 & 7 \\
\hline
\end{tabular}

$n=$ number of cultures

Table 2 E. coli and Enterococcus sp. in urethral flora

\begin{tabular}{|c|c|c|}
\hline \multirow[b]{2}{*}{ Subjects } & \multicolumn{2}{|c|}{ Proportion of positive cultures } \\
\hline & Enterococcus & E. coli \\
\hline Female SCI $(n=17)$ & $0.41^{*}$ & $0.36^{*}$ \\
\hline Bacteriuric & $0.75^{*}$ & $0.71 *$ \\
\hline Non-bacteriuric & $0.31 *$ & $0.28 *$ \\
\hline Normal female $(n=19)$ & 0.37 & 0.26 \\
\hline
\end{tabular}

*Mean proportion of positive cultures bacteriuria is shown in Table 4. E. coli and Enterococcus $\mathrm{sp}$ were the predominant isolates from the urine. Relatively few Klebsiella sp. and no Pseudomonas were isolated from the urine. The same species was not cultured from the urethra in six of $17(33 \%)$ episodes of bacteriuria with E. coli or Enterococcus sp.

The effect of antibiotics on the number of isolates and log numbers of aerobic flora in urethra

Antibiotics did not alter the log numbers of $E$. coli and Enterococci in the urethra but did significantly reduce the total aerobic flora (Table 5). Antibiotics may also have altered other gram negative bacilli in the urethra. Pseudomonas aeruginosa was only isolated from the urethra of patients who had been receiving antibiotics and $E$. coli were not cultured from the urethral swabs of patients who had received quinolones within one week of culture of the urethra.

The bacterial flora number of isolates or log number of bacteria) was not influenced by age, level of injury, or self IC.

Table 3 Mean $\log$ no. E. coli and Enterococcus sp. in urethral flora

\begin{tabular}{lcc}
\hline Subjects & Enterococcus & E. coli \\
\hline Female SCI & & \\
Bacteriuric & $2.49 \pm 0.68(9)^{*}$ & $2.85 \pm 0.69(10)$ \\
& $P<0.05^{* *}$ & $P<0.0001$ \\
Non-bacteriuric & $1.09 \pm 0.29(39)$ & $0.65 \pm 0.20(38)$ \\
& NS & NS \\
Female controls & $1.24 \pm 0.39(19)$ & $0.54 \pm 0.25(19)$ \\
\hline
\end{tabular}

$*()=$ number of observations. ${ }^{* *}=$ Student $t$ test

Table 4 Gram negative bacteria and Enterococcus in the urethra and urine of Patients with bacteriuria

\begin{tabular}{lcc}
\hline Bacteria & Urine & Urethra \\
\hline E.coli & 10 & 7 \\
Klebsiella & 2 & 2 \\
Proteus sp. & 2 & 2 \\
Enterococcus sp. & 9 & 6 \\
S. epidermidis & 3 & ND* $^{*}$ \\
Candida sp. & 1 & ND \\
\hline
\end{tabular}

$* \mathrm{ND}=$ Not done

Table 5 Mean log number of bacteria in the urethra with the use of antibiotics

\begin{tabular}{|c|c|c|c|}
\hline Bacteria & $\begin{array}{c}\text { None } \\
(n=9)\end{array}$ & $\begin{array}{c}\text { Antibiotic } \\
\text { Within } 7 \\
\text { days } \\
(n=8)\end{array}$ & $\begin{array}{c}\text { At time of } \\
\text { culture } \\
(n=12)\end{array}$ \\
\hline E. coli & $0.89 \pm 0.51 *$ & $1.04 \pm 0.59$ & $1.06 \pm 0.35$ \\
\hline Enterococcus & $1.56 \pm 0.54$ & $1.83 \pm 0.51$ & $1.10 \pm 0.46$ \\
\hline Total aerobes & $4.58 \pm 0.33^{* *}$ & $4.33 \pm 0.40$ & $3.40 \pm 0.33^{* *}$ \\
\hline
\end{tabular}

*Mean \pm SDM. ${ }^{* *}$ Student $t$ test. Two tailed $\mathrm{P}$ value $=0.02$ 


\section{Discussion}

Urinary tract infections are a frequent problem in patients with spinal cord injury. At our institution male patients have had a high incidence of infection with Klebsiella and Pseudomonas species which may relate to the use of external condom catheters. ${ }^{3}$ Female patients with SCI tend to have different pathogens. E. coli and Enterococcus sp. accounted for $71 \%$ of infections in female patients receiving intermittent catheterization. ${ }^{1}$ Because of the dominance of E. coli and Enterococcus sp., the bowel has been considered to be the source of the bacteria causing these infections. The pathogenesis of urinary tract infection in women with SCI has been assumed to include contamination of the periurethral area with bacteria from the bowel, colonization of the urethra and entry of the bacteria into the bladder at the time of intermittent catheterization. Our hypothesis was that these patients would be more frequently colonized with larger numbers of aerobic gram negative bacilli and Enterococcus sp. because of bowel incontinence. We were therefore surprised to find that $E$. coli were cultured from the urethral flora of only $36 \%$ of urethral cultures from female patients with SCI and this compared with $26 \%$ of the normal adult women. When isolates at the time of bacteriuria were excluded, E. coli and Enterococcus sp. were isolated in essentially identical proportions and the total number of E. coli and Enterococcus sp. was not different in SCI and normal adult women. Our results suggest that, despite the bowel incontinence, urethral colonization with E. coli or Enterococcus sp. was not increased over that of normal subjects.

Following the initial report of Stamey and his colleagues ${ }^{4}$ many studies have shown that episodes of $E$. coli bacteriuria are usually preceded by colonization of the urethra or periurethra. In the present study we found that bacteriuria with $E$. coli and Enterococcus sp. may be present without simultaneous colonization of the urethra. The E. coli and Enterococcus sp. isolated from the urine was not isolated from the urethra of female patients with SCI in one third of the patients. On four occasions when the same bacteria (E. coli or Enterococcus sp.) was cultured from the urethra and urine, and prior urethral cultures within one week were available, two of the four urethral swabs did not culture the same bacterial species as that found in the urine. Thus as many as two-thirds of the patients with bacteriuria may not have had prior urethral colonization. Our speculation is that in these instances $E$. coli and Enterococcus sp. were residing temporarily in the urethra by contamination and not colonizing the urethral mucosa and that they were inoculated into the bladder with the catheter. Bacteriuria without colonization of the urethra has been recorded in other populations of women. $5-20 \%$ of patients with acute coliform bladder infections have had negative urethral cultures. $^{4-6}$ This may occur considerably more frequently in women with SCI receiving IC and it does raise a question of pathogenicity of these strains. Bacteria that can multiply in the urine and cause bacteriuria but not result in urethral colonization despite repeated bathing of the mucosa with high numbers of organisms may be less pathogenic. E. coli or Enterococcus sp. unable to adhere to the urethral mucosa may also adhere less readily to the mucosa at all levels of the urinary tract. Patients with acute pyelonephritis with $E$. coli have more adhesions than patients with asymptomatic bacteriuria ${ }^{7,8}$ and attachment enhances the virulence of $E$. coli for the urinary tract presumably because it enhances bacterial persistence and invasion of the mucosa. Conversely it has been suggested that bacteria without adherence factors may be nonpathogenic and colonize the urinary tract without symptoms or deterioration of renal function. ${ }^{9}$ We attempted to correlate urethral colonization with symptoms as evidence of pathogenicity. However because of the nature of this study there were insufficient episodes of infection with symptoms to examine this question. Further studies could examine the possibility that a positive urethral culture associated with bacteriuria is an indicator of the pathogenicity of the bacteria causing infections in women with SCI receiving IC.

The use of antibiotics was associated with a reduction in the log number of aerobic bacteria in the urethral flora of the female patients with SCI which may reflect the susceptibility of these organisms to the antibiotics used. The bacterial flora colonizing the periurethral area of normal female subjects, includes S. epidermidis, Lactobacillus sp. Corynebacteria sp. Bacteroides sp. and streptococci. ${ }^{10,11}$ These bacteria could play a role in bacterial interference and account for changes in the gram negative flora. Although we observed no significant effect of antibiotics on the $\log$ numbers of $E$. coli or Enterococci, the presence of aerobic gram negative bacilli in the urethra of patients with SCI may have been influenced by antibiotics. Pseudomonas sp. were only cultured from persons receiving antibiotics. Since the gram negative bacilli cultured from the urethra were most likely from the fecal flora, it is also possible that this observation reflects an indirect effect of antibiotics on the bowel flora that has been described previously. ${ }^{12,13}$

\section{Acknowledgements}

This study was supported by NIH grant HD30506-02.

\section{References}

1 Bennett CJ, Young MN, Darrington H. Differences in urinary tract infections in male and female spinal cord injury patients on intermittent catheterization. Paraplegia 1995; 33: 69-72.

2 Bruce SK et al. Selective media for isolation of Klebsiella pneumoniae. J Clin Microbiol 1981; 13: 1114-1116. 
3 Gilmore DS, Schick DG, Young MN, Montgomerie JZ. Effect of external urinary collection system on colonization and urinary tract infections with Pseudomonas and Klebsiella in men with spinal cord injury. J Am Paraplegic Soc 1992; 15: 206-208.

4 Stamey T, Timothy M, Millar M, Mihara G. Recurrent urinary infections in adult women. The role of introital enterobacteria. Calif Med 1971; 115: 1 - 19.

5 Harding GKM, Ronald AR. A controlled study of antimicrobial prophylaxis of recurrent urinary infection in women. $N$ Engl $J$ Med 1974; 291: 597-601.

6 Stamm WE et al. Diagnosis of coliform infection in acutely dysuric women. $N$ Engl J Med 1982; 307: $463-8$.

7 Svanborg-Eden $\mathrm{C}$ et al. Variable adherence to normal human urinary tract epithelial cells of Escherichia coli strains associated with various forms of urinary tract infections. Lancet 1976; ii: $490-492$.
8 Svanborg-Eden C et al. Host-parasite interaction in the urinary tract. J Infect Dis 1988; 157: 421 - 426.

9 Tencer J. Asymptomatic bacteriuria - a long-term study. Scand J Nephrol 1988; 22: $31-35$.

10 Pfau A, Sacks T. The bacterial flora of the vaginal vestibule, urethra and vagina in the normal premenopausal woman. $J$ Urol 1977; 118: $282-295$.

11 Bartlett JG et al. Quantitative bacteriology of the vaginal fora. $J$ Infect Dis 1977; 136: $271-277$.

12 Daikos $\mathrm{G}$ et al. Comparison of Ciprofloxacin and beta-lactam antibiotics in the treatment of urinary tract infections and alteration of fecal flora. Am J Med 1987; 82: 290-294.

13 Lidefelt KJ, Bollgren I, Nord CE. Changes in periurethral microflora after antimicrobial drugs. Arch Dis Child 1991; 66: $683-685$. 